exciting. It supports nerve-cell attachment, neurite outgrowth, and the establishment of new functional connections between nerve cells that allow the nerve cells to communicate with each other. These are important considerations for the design of new materials for repairing the damaged nervous system."

Unlike other synthetic materials, these peptides are completely biological, composed of amino acids. And unlike parts of animal cells such as collagen that can be extracted as a basis for growing cells but may also carry and pass viruses to the attached growing cells, the peptides do not evoke an immune response or inflammation in living animals.

According to the researchers, while synthetic scaffolds have been used to grow skin, liver tissue, and cartilage, little progress has been made on developing biomaterials for the generation of nerve cells.

\section{With Shrinking Size, Smart \\ Materials Lose their Effectiveness}

Piezoelectric ceramics are commonly used in pressure sensors, microphones, and accelerometers. Deposited as thin films, the material can be used to form sen- sors and actuators in microelectromechanical system (MEMS) devices, elements in ultrasonic motors, and switching capacitors for integrated circuitry. While thin films have much better mechanical properties than the bulk ceramics-for example, films are far less brittle-Nancy Sottos and her research team at the University of Illinois at Urbana-Champaign have found that other physical and electrical properties change in undesirable ways, such as decreases in both piezoelectric response and dielectric constant.

As reported in the April 15 issue of the Journal of Applied Physics, Sottos, a professor of theoretical and applied mechanics, and graduate research assistant Lei Lian obtained lead-zirconate-titanate thin films that ranged in thickness from $0.5 \mu \mathrm{m}$ to $2.0 \mu \mathrm{m}$. To record the films' displacements, Sottos and Lian developed a highresolution, laser Doppler heterodyne interferometric technique.

Sottos said, "The properties of piezoelectric films are critical to the quality and the reliability of MEMS devices. To optimize the performance of thin-film structures, we must first understand the factors that influence those properties."

For their experiments, the measure- ment scheme is based on the Doppler shift. First, the beam from an argon laser strikes a $40-\mathrm{MHz}$ acousto-optic modulator, which produces two beams and sends them along different arms of the interferometer. One beam then bounces off the sample, while the other beam serves as a reference. When the two beams are recombined, the researchers accurately extract the displacement signal from the Doppler shift riding on top of the $40-\mathrm{MHz}$ carrier. The experiments show that as the films become thinner and thinner, their piezoelectric response and dielectric constant decrease. For instance, for films with (111) preferred orientation, as the thickness drops from 2.0 to $0.5 \mu \mathrm{m}$, the dielectric constant drops $\sim 67 \%$ and the $d_{33}$ piezoelectric coefficient drops $\sim 73 \%$.

Significant stresses build up in piezoelectric thin-film structures during the fabrication process, Sottos said. "Changes in the residual-stress state might be one major cause for the change in properties with film thickness that we observed. By applying a mechanical stress-to relieve some of the residual stress- the response of the film can be greatly enhanced."

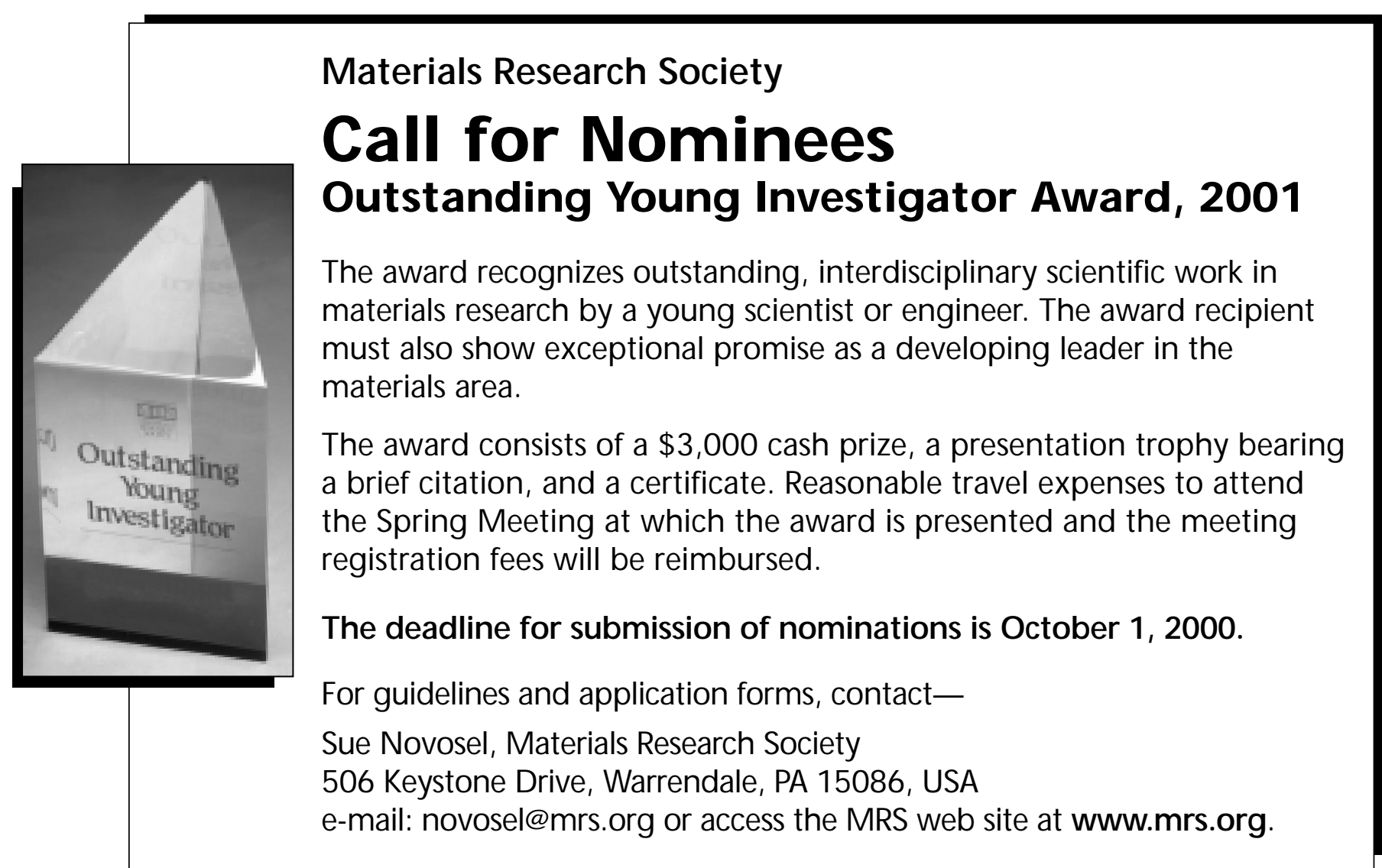

